\section{Diet linked to post-resection colorectal cancer recurrence}

standard chow diet for 4 weeks before colorectal resection surgery. Previous work had demonstrated that anastomoses can attract collagenase-producing microbiota such as Enterococcus faecalis, which might then render these tissues susceptible to tumour formation. Thus, after surgery, all mice received enemas of $E$. faecalis and colon carcinoma cells before colons were removed and analysed 21 days after surgery. Some mice also received prophylactic antibiotics or a collagenase-suppressing compound (Pi-PEG).

Of the mice exposed to E. faecalis, $88 \%$ fed a WD had CRC tumour formation compared with $30 \%$ fed the standard diet. Tumour formation in anastomotic tissues correlated with the presence of E. faecalis and Proteus mirabilis, another collagenolytic bacterial species, but no significant difference was observed between mice fed a WD with or without antibiotics. Although broad antibiotic treatment eliminated the collagenolytic species, the dysbiosis resulted in the emergence of a collagenolytic fungal species. However, administration of Pi-PEG reduced collagenolytic bacteria and tumour formation while maintaining microbial diversity.

"We found that in certain environmental conditions, such as a preoperative high-fat Western diet, and intestinal colonization with collagenase-producing bacteria, tumour cells are promoted to escape the intraluminal environment to form a tumour," concludes Shogan. "We are now trying to understand how collagenolytic organisms promote tumour recurrence and develop novel strategies to prevent it."

Jordan Hindson

ORIGINAL ARTICLE Gaines, S. et al. Western diet promotes intestinal colonization by collagenolytic microbes and promotes tumor formation following colorectal surgery. Gastroenterology https://doi.org/ 10.1053/j.gastro.2019.10.020 (2019)

\title{
LIVER
}

\section{Multispecies liver-on-a-chip for improved drug toxicity testing}

A new study has developed multispecies liver-on-a-chip models (liver-chips) to address challenges in drug development such as species-specific differences in drug metabolism and toxicity. This technology could lead to better predictions of liver toxicities to determine human risk.

Before moving into human clinical trials, new drug candidates must undergo safety evaluation in animal models, typically rat and dog. However, toxicities in humans are not always correctly predicted with these models, particularly those related to druginduced liver injury (DILI), or the results from different models might be discordant. This situation leads to drug failures in trials and the clinic, and to substantial costs for drug development, highlighting a need for better predictive alternatives.

One solution is organ-on-chip technology, which recreates the 3D microenvironment and function of organs.
In collaboration with pharmaceutical companies, Donald Ingber and colleagues at Emulate led by Geraldine Hamilton, who have previously worked on several organ-on-chip models, aimed to develop rat, dog and human liver-chips to address these challenges.

Microfluidic liver-chips were microengineered to contain speciesspecific primary hepatocytes and liver sinusoidal endothelial cells, with or without Kupffer cells and/or hepatic stellate cells, and all cultured under physiological fluid flow. After assessing physiological function and relevance of the liver-chips, the investigators explored prediction of species-specific DILI responses. The liver-chips successfully detected DILI phenotypes including hepatocellular injury, steatosis, cholestasis and fibrosis.

"We confirmed that rat, dog, and human liver-chips recapitulated species-specific toxicities that were also observed in previous animal and

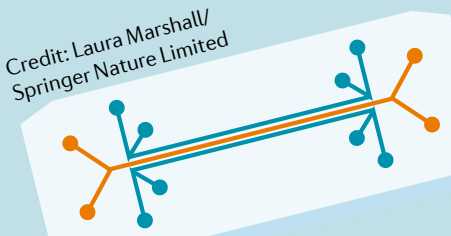

human studies by our pharma partners," reports Ingber. "In addition, because we can carry out high-resolution imaging in our chips, we uncovered a potential mechanism of toxicity of one compound that was not previously appreciated," he says.

In tests of other experimental compounds, one induced fibrosis in the rat liver-chip but did not alter function of the human liver-chip, demonstrating species-specific differences. "The hope in the future is that if the human liverchips can be further validated in terms of their mimicry of human hepatotoxicities relative to animal models, this could progressively reduce, and eventually replace, the use of animals in toxicity studies," concludes Ingber.

lain Dickson

ORIGINAL ARTICLE Jang, K.-J. et al. Reproducing

human and cross-species drug toxicities using a Liver-Chip. Sci. Transl Med. 11, eaax5516 (2019) 